\title{
Serum microRNA-155 as a potential biomarker for breast cancer screening
}

\author{
ZHAO SuYing ${ }^{1}$, WU Qian ${ }^{2}$, GAO Fen ${ }^{1}$, ZHANG ChunBing $^{1 *} \&$ YANG XueWen $^{1}$ \\ ${ }^{1}$ Department of Laboratory Medicine, Jiangsu Provincial Hospital of TCM, Nanjing 210029, China; \\ ${ }^{2}$ School of Public Health, Nanjing Medical University, Nanjing 210029, China
}

Received October 24, 2011; accepted April 15, 2012

\begin{abstract}
MicroRNAs (miRs) have been shown to be differentially expressed in the serum of cancer patients and controls, and can thus be used as biomarkers for cancer screening. We detected the expression level of miR-155 in the serum of female breast cancer patients and healthy controls to investigate whether serum miR-155 could discriminate patients with early-stage breast cancer. Serum samples were collected from 20 female patients with newly diagnosed breast cancer and 10 healthy controls. Real-time quantitative PCR was used to detect the expression level of miR-155. The expression level of miR-155 was significantly increased in the serum of breast cancer patients compared with in the serum of normal controls. MiR-155 may be useful as a blood-based biomarker for breast cancer screening.
\end{abstract}

microRNA, miR-155, breast cancer, serum, biomarker

Citation: Zhao S Y, Wu Q, Gao F, et al. Serum microRNA-155 as a potential biomarker for breast cancer screening. Chin Sci Bull, 2012, 57: 3466-3468, doi: $10.1007 /$ s11434-012-5362-1

Breast cancer is the second most common cancer in women worldwide, and accounts for approximately $7 \%-10 \%$ of all malignant tumors. Its incidence is second only to uterine cancer [1]. Most women with breast cancer are diagnosed at an advanced disease stage because its early symptoms are insidious; screening for breast cancer allows early diagnosis and potentially reduces mortality. However, because of the absence of symptoms, palpation is currently the most reliable screening tool for breast cancer. MicroRNAs (miRNAs) are small non-protein-coding RNA sequences of approximately 19 to 26 nucleotides [2]. The first known microRNA, lin-4, was discovered in 1993 through a study of the heterochronic gene lin-14 in worms [3]. Since then, miRNAs have been shown to play important regulatory roles in many biological and pathological processes. Their detailed mechanism of action is still under debate, but is probably related to inhibition of translation and mRNA degradation [4]. MicroRNAs regulate gene expression at the level of translation through imperfect pairing with target mRNAs of protein-

*Corresponding author (email: zhang_chb99@yahoo.com.cn) coding genes [5]. There is increasing evidence suggesting that some miRNAs are linked to human oncogenesis; this has revealed new opportunities for non-invasive tests for early cancer diagnosis [6]. Iorio et al. [7] compared the expression levels of miRNAs in 76 primary breast cancer tissues and 10 normal tissues and found significant downregulation of miR-125b, miR-145, miR-21, and miR-155 in cancer tissues [7]. Mitchell et al. [8] demonstrated that miRNAs are present in human plasma and serum in a remarkably stable form. This makes it possible to use serum miRNAs as non-invasive biomarkers for the detection of cancer. Here, we quantified the expression level of miR-155 in the serum of female breast cancer patients and healthy controls, and evaluated the feasibility of using miR-155 as a non-invasive biomarker for early-stage breast cancer screening.

\section{Materials and methods}

\subsection{Sample collection and serum processing}

Informed consent was obtained from the participants in this 
study. Blood samples were collected from 30 women including 20 breast cancer patients and 10 healthy subjects as normal controls, aged between 35 and 65 years old. The participant characteristics and the distribution of cancer stages in accordance with pathology microscopy are summarized in Table 1. Blood was collected from the antecubital fossa and placed in a serum separator tube. The collected blood was allowed to stand for no more than $1 \mathrm{~h}$ at room temperature before being centrifuged at $2465 \times g$ for 5 min to obtain serum [1]. The resultant serum was aliquoted into Eppendorf tubes and stored at $-80^{\circ} \mathrm{C}$.

\subsection{RNA extraction}

Total RNA, which included miRNAs, was isolated from serum using an miRNeasy Mini kit (Qiagen, USA) following the manufacturer's instructions. Next, $400 \mu \mathrm{L}$ of Trizol LS reagent (Qiagen) was added to $200 \mu \mathrm{L}$ of serum sample. After phase separation by chloroform addition, the samples were centrifuged for $15 \mathrm{~min}$ at $12000 \times g$ at $4^{\circ} \mathrm{C}$. A $1.5 \times$ volume of $100 \%$ ethanol was added to the upper aqueous phase, and $700 \mu \mathrm{L}$ of the sample was pipetted into an RNeasy Mini column (Qiagen). The RNA was eluted by RNase-free water in a final volume of $30 \mu \mathrm{L}$ and was stored at $-80^{\circ} \mathrm{C}$ until use.

\subsection{Reverse transcription (RT) and real-time quantita- tive PCR (qPCR)}

Total RNA extracted from serum was reverse transcribed to cDNA using a MiScript Reverse Transcription kit (Qiagen) according to the manufacturer's protocol with the following modifications: each reaction was performed in a final volume of $20 \mu \mathrm{L}$ containing $4 \mu \mathrm{L}$ of total RNA, $4 \mu \mathrm{L}$ of RT buffer and $1 \mu \mathrm{L}$ of RTase mix. The reverse transcription profile was $37^{\circ} \mathrm{C}$ for $60 \mathrm{~min}$ and $95^{\circ} \mathrm{C}$ for $5 \mathrm{~min}$, after which the sample was maintained at $4^{\circ} \mathrm{C}$. Real-time qPCR was performed using a MiScript SYBR Green PCR kit (Qiagen) and Assay miR-155 (Qiagen). Two microliters of cDNA were used as a template in a $20-\mu \mathrm{L}$ reaction with the following conditions: denaturation at $95^{\circ} \mathrm{C}$ for $15 \mathrm{~min}$ followed by 40 cycles of $94^{\circ} \mathrm{C}$ for $15 \mathrm{~s}, 55^{\circ} \mathrm{C}$ for $30 \mathrm{~s}$, and $70^{\circ} \mathrm{C}$ for $34 \mathrm{~s}$, in which fluorescence was acquired and detected by an ABI PRISM 7500 Real-time PCR system

Table 1 Participant characteristics

\begin{tabular}{lcc}
\hline & Breast cancer patients $(n=20)$ & Healthy controls $(n=10)$ \\
\hline Age (years) & & \\
Mean & 54 & 51 \\
Range & $45-64$ & $35-65$ \\
\hline Disease stage & & \\
I & 1 & - \\
II & 19 & - \\
\hline
\end{tabular}

(Applied Biosystems, USA). After the PCR cycles, melting curve analyses were performed to validate the specific generation of the expected PCR product. As there is no known control miRNA in serum, we used RNU6B as an endogenous control, because a study has shown that RNU6B is readily detectable in the serum and displayed little variability in expression levels between individuals [9]. We normalized the expression levels of miR-155 to those of RNU6B. The MiScript primer sequence for miR-155 was 5'-CUCCUACAUAUUAGCAUUAACA-3'. The expression level of miR-155 was evaluated using the $\Delta C t$ method. The cycle threshold $(C t)$ value is the number of qPCR cycles required for the fluorescent signal to cross a specified threshold. $\Delta C t$ was calculated by subtracting the $C t$ values of RNU6B from those of miR-155. $\Delta \Delta C t$ was calculated by subtracting the $\Delta C t$ of the control samples from the $\Delta C t$ of the cancer samples.

\subsection{Statistical analysis}

The fold change in miR-155 expression was calculated by the equation $2^{-\Delta \Delta C t}$. The significance of the change was determined using the Mann-Whitney test. All $P$ values less than 0.05 were considered statistically significant. Data were analyzed using the statistical software SPSS v.17.0 (SPSS, Inc, Chicago, IL, USA).

\section{Results}

Parts of the amplification curves are shown in Figure 1. The expression level of miR-155 was significantly up-regulated in serum from breast cancer patients compared with in serum from healthy controls $(P<0.05$; Figure 2$)$.

\section{Discussion}

Until recently, miRNAs were thought to be unstable in the peripheral circulation and were termed "tissue-based tumor markers". However, in 2008, Mitchell et al. [8] demonstrated that miRNAs are present in human plasma and serum in a remarkably stable form that is protected from endogenous RNase activity. The efficient extraction and accurate identification of miRNAs from serum represents a key first step towards the development of a non-invasive, blood-based detection test for breast cancer. This report describes a quantitative assessment of serum miR-155 levels in female breast cancer patients. We used miR-155 because it had been previously shown to be down-regulated in breast cancer [7]; however our data contradict those of the previous study because we identified significantly elevated levels of miR-155 in the serum of breast cancer patients. Instead, our results are concordant with those of another study [10]. It is possible that the conflicting results are 


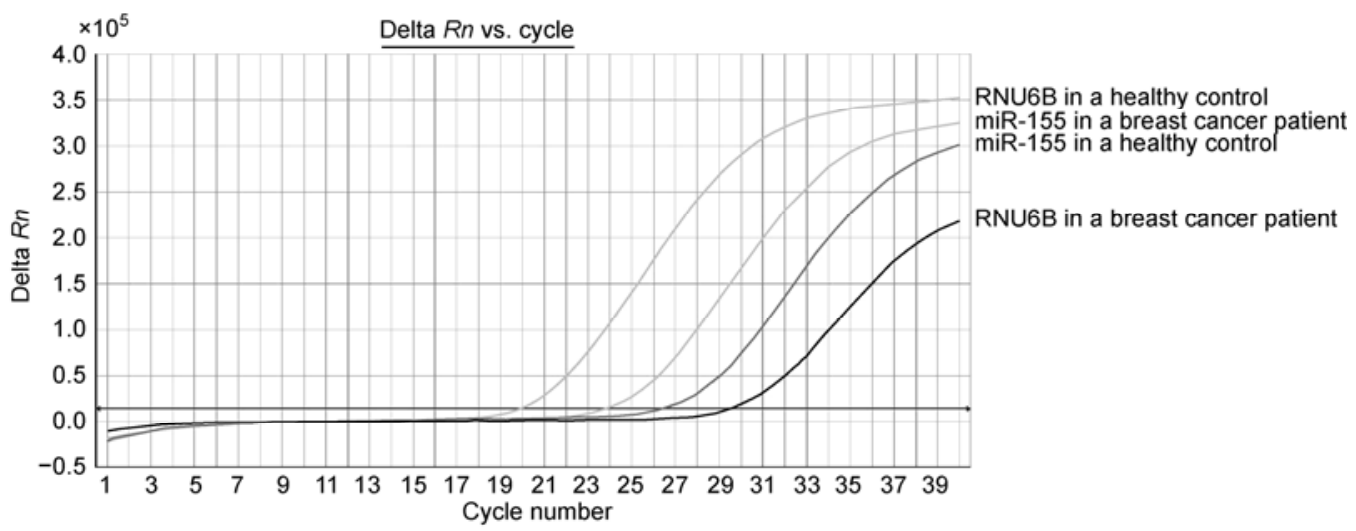

Figure 1 Amplification curves of miR-155 and an RNU6B control in the serum of breast cancer patients and healthy controls.

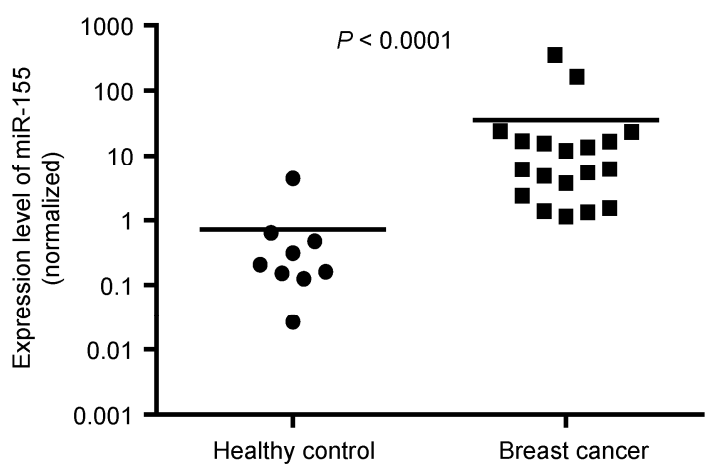

Figure 2 Scatter plot of the expression level of miR-155 in the serum of breast cancer patients and healthy controls. There was a statistically significant difference between the median expression levels (horizontal lines).

because of different miRNA expression levels in different organs and sample types, although we must also consider that our sample size was small, which may have influenced our conclusions.

We have shown that microRNAs are stable in the peripheral circulation and could be used as biological markers for breast cancer detection. The breast cancer patients chosen for this study were all in early metaphase; this implies that miR-155 can be used in the early diagnosis of breast cancer. Tumorigenesis is a complicated process that varies in different individuals over time; as our sample size was very small, further validation of the usefulness of this marker in large cohorts and in independent studies is necessary. It is likely however, that using a single serum miRNA as a biomarker for breast cancer screening will be insufficient. Detecting multiple miRNAs together with other tumor markers, for example protein antigens, will be necessary for accurate diagnosis. It will also be important to investigate the mechanisms of miRNA function in the different stages of carcinogenesis and among different individuals [11]. Nonetheless, the availability of powerful yet simple approaches for serum miRNA detection and quantification suggests that miRNAs have great potential as biomarkers. In addition, the inherent regulatory function of miRNAs implies that those expressed in tumors may influence the biological behavior and clinical phenotype of that tumor [12]. In other words, serum miRNAs may serve as biomarkers not only for diagnosis but also to predict clinical behavior and/or therapeutic response.

This work was supported by the Jiangsu Provincial Hospital of TCM.

1 Schaeffer P, Mulcahy H E, Meyer P, et al. Telomerase RNA as a detection marker in the serum of breast cancer patients. Clin Cancer Res, 2000, 6: 3823-3826

2 Lee R C, Ambros V. An extensive class of small RNAs in Caenorhabditis elegans. Science, 2001, 294: 862-864

3 Lee R C, Feinbaum R L, Ambros V. The C. elegans heterochronic gene lin-4 encodes small RNAs with antisense complementarity to lin-14. Cell, 1993, 75: 843-854

4 Calin G A, Croce C M. MicroRNA signatures in human cancers. Nat Rev Cancer, 2006, 6: 857-866

5 Kim V N, Nam J W. Genomics of microRNA. Trends Genet, 2006, 22: $165-173$

6 Sotiropoulou G, Pampalakis G, Lianidou E, et al. Emerging roles of microRNAs as molecular switches in the integrated circuit of the cancer cell. RNA, 2009, 15: 1443-1461

7 Iorio M V, Ferracin M, Liu C G, et al. MicroRNA gene expression deregulation in human breast cancer. Cancer Res, 2005, 65: 70657070

8 Mitchell P S, Parkin R K, Kroh E M, et al. Circulating microRNAs as stable blood-based markers for cancer detection. Proc Natl Acad Sci USA, 2008, 105: 10513-10518

9 Ng E K, Chong W W, Jin H, et al. Differential expression of microRNAs in plasma of colorectal cancer patients: A potential marker for colorectal cancer screening. Gut, 2009, 58: 1375-1381

10 Zhu W Z, Qin W Y, Ulus A, et al. Circulating microRNAs in breast cancer and healthy subjects. BMC Res Notes, 2009, 2: 89

11 Chen X, Ba Y, Ma L, et al. Characterization of microRNAs in serum: A novel class of biomarkers for diagnosis of cancer and other diseases. Cell Res, 2008, 10: 1038

12 Israel A, Sharan R, Ruppin E, et al. Increased microRNA activity in human cancers. PLoS One, 2009, 4: 6045

Open Access This article is distributed under the terms of the Creative Commons Attribution License which permits any use, distribution, and reproduction in any medium, provided the original author(s) and source are credited. 